\title{
Construção Qualitativa sobre o Portfólio Bibliográfico com tema "Radiação Térmica em Argamassas de Revestimento"
}

\author{
Géssica Katalyne Bilcatil'; Marienne do Rocio de Mello Maron da Costa'; Franciele Bonatto \\ Elaine Cristina Lengowsk $\dot{p}^{3}$ \\ 凹elainelengowski@gmail.com
}

1. Universidade Tecnológica Federal do Paraná, PR - Brasil. 2. Universidade Federal do Paraná, PR - Brasil. 3. Universidade Federal do Mato Grosso, MT - Brasil.

Histórico do Artigo:

Recebido em: 18 de julho de 2019 Aceito em: 09 de dezembro de 2019 Publicado em: 28 de dezembro de 2019

Resumo: A avaliação do desempenho térmico de paredes de uma edificação é de suma importância uma vez que as condições de conforto térmico refletem diretamente na qualidade de moradia dos usuários. Deste modo, o objetivo deste artigo é analisar como o tema radiação térmica foi abordado e quais os métodos utilizados pelas pesquisas anteriores para avaliar a radiação térmica em argamassas no segmento da construção civil. Dessa forma, foi realizada uma revisão sistemática da literatura, a nível internacional, dos últimos 10 anos, utilizando a metodologia MethodiOrdinatio. Com base no estudo dos artigos foi concluído que o estudo de radiação térmica em argamassas de revestimento teve um crescimento significativo no ano de 2017. A radiação térmica geralmente é negligenciada nos cálculos de desempenho térmico, não sendo considerada nas normas de muitos países. 0 método da câmera de termografia por infravermelho como ferramenta para análise do desempenho térmico, tem sido amplamente utilizado em análises qualitativas em elementos de construção. No entanto, para estudos quantitativos, os ensaios por termografia por infravermelho ainda são insipientes.

Palavras-chave: MethodiOrdnatio; Desempenho térmico; Avaliação sistemática.

\section{Qualitative Construction on The Bibliographic Portfolio with theme "Thermal Radiation in Coating Mortars"}

Abstract: The evaluation of the thermal performance of walls of a building is of paramount importance since the thermal comfort conditions reflect directly on the quality of housing of users. Thus, the objective of this paper is to analyze how the thermal radiation theme was approached and which methods were used by previous research to evaluate the thermal radiation in mortars in the civil construction segment. Thus, a systematic review literature of the last 10 years was carried out using the MethodiOrdinatio methodology. Based on the study of the articles it was concluded that the study of thermal radiation in coating mortar had significant growth in the year 2017. Thermal radiation is generally neglected in thermal performance calculations and is not considered in many countries standards. The infrared thermography camera method as a tool for thermal performance analysis has been widely used in qualitative analysis of building elements. However, for quantitative studies, infrared thermography assays are still incipient.

Keywords: MethodiOrdnatio; Thermal performance; Systematic evaluation. 


\section{Construcción Cualitativa de la Cartera Bibliográfica con el tema "La Radiación Térmica en los Morteros de Recubrimiento"}

Resumen: La evaluación del rendimiento térmico de las paredes de un edificio es de suma importancia ya que las condiciones de confort térmico se reflejan directamente en la calidad de la vivienda de los usuarios. Por lo tanto, el objetivo de este trabajo es analizar cómo se abordó el tema de la radiación térmica y qué métodos fueron utilizados por investigaciones anteriores para evaluar la radiación térmica en morteros en el segmento de la construcción civil. Por lo tanto, se realizó una revisión sistemática de la literatura de los últimos 10 años utilizando la metodología MethodiOrdinatio. Según el estudio de los artículos, se concluyó que el estudio de la radiación térmica en el mortero de revestimiento tuvo un crecimiento significativo en el año 2017. La radiación térmica generalmente se descuida en los cálculos de rendimiento térmico y no se considera en los estándares de muchos países. El método de cámara termográfica infrarroja como herramienta para el análisis del rendimiento térmico se ha utilizado ampliamente en el análisis cualitativo de elementos de construcción. Sin embargo, para estudios cuantitativos, los ensayos de termografía infrarroja aún son incipientes.

Palabras clave: MethodiOrdnatio; Rendimiento térmico; Evaluación sistemática.

\section{INTRODUÇ̃̃o}

0 estudo do desempenho térmico é essencial tanto no que diz respeito à redução de impactos ambientais quanto à melhoria da qualidade de vida e engloba a resposta do projeto arquitetônico proposto em relação às trocas térmicas entre 0 ambiente construído e 0 ambiente externo, sendo que a caracterização das condições climáticas locais é uma das etapas mais importantes do projeto (PIZZUTI et al., 2016).

0 cálculo teórico para avaliação do desempenho térmico é essencial durante a fase de projeto de uma obra, porém o resultado final do desempenho térmico pode variar devido a irregularidades de construção, relacionadas a qualidade do acabamento, fluxo de calor, umidade e propriedades inerentes ao material e seus efeitos de degradação. Devido a isso a medição in loco, pode ser necessária para pós-avaliação do desempenho térmico da construção (ATSONIOS et al., 2017).

Os sistemas de paredes de construção são os principais responsáveis pela regulação do conforto térmico em resposta às condições climáticas externas e atuam como uma interface que separa o ambiente externo do ambiente interno. 0 comportamento térmico de um material de construção depende das condutividades térmicas das partículas sólidas, da microestrutura do poro e do seu fluido constituinte (ar e/ou umidade), em que a transferência de calor (condução, convecção e radiação) é influenciada por redes complexas de poros e partículas (BALAJI et al., 2014).

As características de desempenho térmico com base em testes de componentes de construção em condições dinâmicas reais ajudam a preencher a lacuna entre o desempenho 
teoricamente previsto e o desempenho prático das edificações. 0s métodos de ensaios para avaliação do desempenho térmico exigem resultados que demonstrem o comportamento real das edificações, tanto no ambiente de teste como na configuração experimental, bem como os métodos de análise de dados dinâmicos para chegar a uma caracterização com precisão confiável (ROELS et al., 2015).

A avaliação do desempenho térmico é necessária para a compreensão de técnicas empregadas na construção civil para benefício do conforto térmico das pessoas e da eficiência energética das edificações nas diferentes regiões, com condições climáticas variadas. A energia utilizada para o resfriamento e aquecimento é uma parte importante das contas de energia em regiões climáticas extremamente quentes e frias, pois a maioria dessas casas precisam de uma reabilitação para ser adaptável com os requisitos contemporâneos de conforto (SASSINE et al., 2017).

A transferência de calor através da radiação solar tem grande influência no comportamento térmico das paredes de construção e é muitas vezes ignorada nos cálculos de desempenho térmico das edificações. A Norma europeia, assim como a Norma brasileira, não prevê a transferência de calor devido à radiação térmica. As propriedades térmicas, especialmente a condutividade térmica são dependentes do clima incluindo umidade e radiação solar. Recentemente alguns pesquisadores começaram a estudar as propriedades térmicas com base em medições in loco, como temperaturas da superfície e fluxos de calor (KIRIMTAT; KREJCAR, 2018).

A norma de desempenho NBR 15575 (2013) exige parâmetros de desempenho térmico para edificações na qual se recomenda dois métodos para análise: método simplificado e método de simulação computacional. 0 método simplificado exige que os projetos cumpram requisitos prescritivos de elementos de vedações verticais e coberturas, sendo que para cada zona bioclimática brasileira são exigidos requisitos máximos de transmitância térmica (Uvalue) e valores mínimos de capacidade térmica (CT). Caso um projeto não atenda aos índices prescritivos para transmitância térmica e capacidade térmica de sua envoltória previstos pelo método simplificado, ele deve ser submetido a uma simulação computacional de desempenho, no qual é elaborado um modelo computacional do edifício, com sua orientação solar, volumetria e materiais de envoltória (LAMBERTS, 2014).

A finalidade da norma NBR 15575 (2013) é representar a produção de edifícios com melhores níveis de conforto e menor consumo de energia. Existem, no entanto, deficiências na estrutura da norma e em seus critérios de análise, bem como limitações nos padrões atuais 
Construção Qualitativa sobre o Portfólio Bibliográfico com tema "Radiação Térmica em Argamassas de Revestimento"

devido a diferentes aspectos: arquivos meteorológicos, falta de consideração de estratégias passivas e inconsistência com o método simplificado, permitindo que edifícios termicamente ineficientes sejam projetados e construídos dentro dos parâmetros desta norma (MARQUES e CHVATAL, 2013).

Considerando a relevância do estudo da radiação térmica no sistema construtivo, este artigo tem como objetivo analisar como o tema radiação térmica foi estudado e quais os métodos utilizados pelas pesquisas anteriores para avaliar a radiação térmica em argamassas no segmento da construção civil. Para alcançar o objetivo, foi realizada através da metodologia MethodiOrdinatio, uma revisão sistemática da literatura entre 2008 e 2018 em cinco bases de dados.

\section{MATERIAL E MÉTODOS}

Trata-se de uma pesquisa usando a metodologia MethodiOrdinatio, que resulta em um portfólio bibliográfico abrangendo as pesquisas mais relevantes sobre o tema (PAGANI et al., 2015). Para aplicação deste método, as seguidas as fases são seguidas.

Na fase 1 foram estudadas a intenção de pesquisa. Para 0 artigo estudado, a intenção da pesquisa foi "Radiação Térmica em Argamassas de Revestimento"; o eixo definido para proceder à pesquisa em bases de dados foi "Thermal radiation" AND "Mortar". 0 limite temporal estabelecido para a pesquisa foi de 2008 a 2018. Em relação às bases de dados, foram selecionados aquelas que apresentaram maior número de trabalhos pesquisados, sendo elas: Science Direct, Springer, AIP, Web of Science e Scopus.

Na fase 2 foi feita a pesquisa final em bancos de dados usando o gerenciador de referência, Mendeley. A Tabela 1 mostra a volume de artigos obtidos considerando cada base de dados pesquisada.

Tabela 1. Artigos obtidos em cada base de dados.

Bases de dados

\begin{tabular}{|c|c|c|c|c|c|}
\hline $\begin{array}{c}\text { Palavras- } \\
\text { chave }\end{array}$ & $\begin{array}{c}\text { Science } \\
\text { direct }\end{array}$ & Springer & Aip & $\begin{array}{l}\text { Web of } \\
\text { science }\end{array}$ & Scopus \\
\hline
\end{tabular}

Total

TOTAL DE ARTIGOS ENCONTRADOS

"Thermal

radiation” 
and mortar

234

112

131

100

53

630

Fonte: 0 s autores.

Na fase 3, os artigos foram analisados individualmente aplicando os seguintes filtros: eliminando a duplicidade usando o gerenciador de referência (Mendeley); artigos cujo título não estava relacionado com a área; artigos pertencentes a capítulos de livro ou conferências devido à ausência do fator de impacto. Para finalizar essa etapa, as palavras-chave e os resumos foram analisados para verificar se o artigo estava ou não relacionado com a área. $\mathrm{Na}$ Tabela 2 é mostrado o procedimento de filtragem.

Tabela 2. Procedimento de filtragem dos artigos estudados.

Procedimentos de filtragem usados $\quad$ Artigos selecionados $\quad$ Artigos
excluídos

Total bruto de artigos 630 100

Artigos de capítulos de livros/ conferências 126 20

Duplicidade de artigos 112 17,78

Número de artigos excluídos após leitura do 312

título

Número de artigos excluídos após leitura do resumo

Total de artigos descartados

605 96,03

Total de artigos selecionados para compor o 25 3,97 portfólio

Fonte: Os autores (2019).

Através do procedimento de filtragem realizado foram selecionados 25 artigos para compor o escopo da pesquisa. 0 próximo passo foi classificar os artigos usando a Equação In0rdinatio (1).

$$
\text { InOrdinatio } \left.=\left(\frac{I F}{1000}\right)+(\alpha \cdot(10-\mathrm{RY}-\mathrm{PY}))\right)+\mathrm{Ci}
$$


Construção Qualitativa sobre o Portfólio Bibliográfico com tema "Radiação Térmica em Argamassas de Revestimento"

Onde: $\mathrm{IF}=$ Fator de Impacto da revista; $\alpha=$ coeficiente de relevância do ano da pesquisa; $\mathrm{RY}=$ Ano da pesquisa; $\mathrm{PY}=$ Ano da publicação; $\mathrm{Ci}=$ números de citações.

Através da fase 4 foi realizada a pesquisa pelo fator de impacto e número de citação, então foi utilizado o gerenciador de referência JabRef, que forneceu automaticamente uma lista de artigos com o nome dos autores, título do trabalho, nome da revista, ano de publicação, tipo de publicação e URL. 0 número de citações foi extraído manualmente de Google Acadêmico. 0 fator de impacto também foi obtido manualmente através da lista fornecida pelo Scopus2.

Na última fase foi obtido o ranking dos artigos usando o In0rdinatio utilizando os parâmetros obtidos na fase 4. 0s 25 artigos foram classificados do maior para o menor Índice Ordinatio (In0rdinatio) usando valores alfa $(\alpha)$ de 10. Sendo o valor de alfa $(\alpha)$, um critério em que 0 pesquisador tem a liberdade de escolher, e até mesmo mudar, durante este passo. 0 valor de alfa $(\alpha)$ igual 10 representa que o ano de publicação do artigo é um fator relevante para a pesquisa (PAGANI et al., 2015).

\section{RESULTADOS E DISCUSSÃ0}

Foram identificados 25 artigos (tabela 3) através do método MethodiOrdinatio, e estes foram ranqueados considerando o número de citações, ano de publicação e fator de impacto da revista.

Tabela 3. Procedimento de filtragem dos artigos estudados.

\begin{tabular}{clcc}
\hline Ranking & \multicolumn{1}{c}{ Autores e título do artigo } & Citações & Ano de publicação \\
\hline $\mathbf{1}^{\mathbf{0}}$ & $\begin{array}{l}\text { Bagavathiappan, S., Lahiri, B., Saravanan, } \\
\text { T., Philip, J. and Jayakumar, T. Infrared } \\
\text { thermography for condition monitoring - } \\
\text { A review. }\end{array}$ & 2013 \\
\hline $\mathbf{2}^{\mathbf{0}}$ & $\begin{array}{l}\text { Kylili, A., Fokaides, P.A., Christou, P. and } \\
\text { Kalogirou, S.A. Infrared thermography } \\
\text { (IRT) applications for building } \\
\text { diagnostics: A review. }\end{array}$ & \\
\hline $\mathbf{3}^{\mathbf{0}}$ & $\begin{array}{l}\text { Fokaides, P.A. and Kalogirou, S.A. } \\
\text { Application of infrared thermography for }\end{array}$ & \\
\hline
\end{tabular}




\begin{tabular}{|c|c|c|c|}
\hline & $\begin{array}{l}\text { the determination of the overall heat } \\
\text { transfer coefficient (U-Value) in building } \\
\text { envelopes. }\end{array}$ & & \\
\hline $4^{0}$ & $\begin{array}{l}\text { Barreira, E., Almeida, R. and Delgado, J. } \\
\text { Infrared thermography for assessing } \\
\text { moisture related phenomena in building } \\
\text { components. Low-emissivity materials for } \\
\text { building applications: A state-of-the-art } \\
\text { review and future research perspectives. }\end{array}$ & 41 & 2016 \\
\hline $5^{0}$ & $\begin{array}{l}\text { Jelle, B. P., Kalnaes, S. E. and Gao, T. Low- } \\
\text { emissivity materials for building } \\
\text { applications: A state-of-the-art review } \\
\text { and future research perspectives. }\end{array}$ & 44 & 2015 \\
\hline $6^{\circ}$ & $\begin{array}{l}\text { Lucchi, E. Applications of the infrared } \\
\text { thermography in the energy audit of } \\
\text { buildings: A review. }\end{array}$ & 13 & 2018 \\
\hline $7^{\circ}$ & $\begin{array}{l}\text { Tong, G., Christopher, D. and Li, B. } \\
\text { Detection of façade plaster detachments } \\
\text { using infrared thermography - A } \\
\text { nondestructive technique. }\end{array}$ & 101 & 2009 \\
\hline $8^{\circ}$ & $\begin{array}{l}\text { Freitas, S.S., de Freitas, V.P. and Barreira, } \\
\text { E. Quantitative internal infrared } \\
\text { thermography for determining in-situ } \\
\text { thermal behaviour of façades. }\end{array}$ & 14 & 2017 \\
\hline $9^{\circ}$ & $\begin{array}{l}\text { Tejedor, B., Casals, M., Gangolells, M. and } \\
\text { Roca, X. A comparative assessment of the } \\
\text { standardized methods for the in-situ } \\
\text { measurement of the thermal resistance } \\
\text { of building walls. }\end{array}$ & 11 & 2017 \\
\hline $10^{\circ}$ & $\begin{array}{l}\text { Atsonios, I.A., Mandilaras, I.D., } \\
\text { Kontogeorgos, D.A. and Founti, M.A. A } \\
\text { review of infrared thermography for the } \\
\text { investigation of building envelopes: } \\
\text { Advances and prospects. }\end{array}$ & 0 & 2018 \\
\hline $11^{\circ}$ & $\begin{array}{l}\text { Lehmann, B.; Dorer, V.; Koschenz, M. } \\
\text { Definition of an experimental procedure } \\
\text { with the hot box method for the thermal } \\
\text { performance evaluation of }\end{array}$ & 67 & 2013 \\
\hline
\end{tabular}




\begin{tabular}{|c|c|c|c|}
\hline & inhomogeneous walls. & & \\
\hline $12^{0}$ & $\begin{array}{l}\text { Alavéz-Ramírez, R., Chiñas-Castillo, F., } \\
\text { Morales-Domínguez, V.J., Ortiz-Guzmán, } \\
\text { M., Caballero-Montes, J.L. and Caballero- } \\
\text { Caballero, M. Thermal lag and decrement } \\
\text { factor of constructive component } \\
\text { reinforced mortar channels filled with } \\
\text { soil-cement-sawdust. }\end{array}$ & 0 & 2018 \\
\hline $13^{\circ}$ & $\begin{array}{l}\text { Briga-Sá, A., Boaventura-Cunha, J., } \\
\text { Lanzinha, J.-C. and Paiva, A. Experimental } \\
\text { and analytical approach on the Trombe } \\
\text { wall thermal performance parameters } \\
\text { characterization. }\end{array}$ & 3 & 2017 \\
\hline $14^{0}$ & $\begin{array}{l}\text { Aversa, P., Palumbo, D., Donatelli, A., } \\
\text { Tamborrino, R., Ancona, F., Galietti, U. and } \\
\text { Luprano, V.A.M. Infrared thermography } \\
\text { for the investigation of dynamic thermal } \\
\text { behaviour of opaque building elements: } \\
\text { Comparison between empty and filled } \\
\text { with hemp fibres prototype walls. }\end{array}$ & 1 & 2017 \\
\hline $15^{\circ}$ & $\begin{array}{l}\text { Yang, W., Zhu, X. and Liu, J. Annual } \\
\text { experimental research on convective heat } \\
\text { transfer coefficient of exterior surface of } \\
\text { building external wall. Numerical } \\
\text { simulation of coupled heat transfer } \\
\text { through double hollow brick walls: Effects } \\
\text { of mortar joint thickness and emissivity. }\end{array}$ & 1 & 2017 \\
\hline $16^{0}$ & $\begin{array}{l}\text { Boukendil, M., Abdelbaki, A. and Zrikem, Z. } \\
\text { Numerical simulation of coupled heat } \\
\text { transfer through double hollow brick } \\
\text { walls: Effects of mortar joint thickness } \\
\text { and emissivity. }\end{array}$ & 1 & 2017 \\
\hline $17^{\circ}$ & $\begin{array}{l}\text { Kanellopoulos, G., Koutsomarkos, V., } \\
\text { Kontoleon, K. and Georgiadis-Filikas, K. } \\
\text { Numerical Analysis and Modelling of Heat } \\
\text { Transfer Processes through Perforated } \\
\text { Clay Brick Masonry Walls }\end{array}$ & 1 & 2017 \\
\hline $18^{\circ}$ & Barrios, G., Casas, J., Huelsz, G. and Rojas, & 5 & 2016 \\
\hline
\end{tabular}




\begin{tabular}{|c|c|c|c|}
\hline & $\begin{array}{l}\text { J. Ener-Habitat: An online numerical tool } \\
\text { to evaluate the thermal performance of } \\
\text { homogeneous and non-homogeneous } \\
\text { envelope walls/roofs. }\end{array}$ & & \\
\hline $19^{\circ}$ & $\begin{array}{l}\text { Naveros, I., Bacher, P., Ruiz, D., Jiménez, } \\
\text { M. and Madsen, H. Setting up and } \\
\text { validating a complex model for a simple } \\
\text { homogeneous wall. }\end{array}$ & 22 & 2014 \\
\hline $20^{\circ}$ & $\begin{array}{l}\text { Li, L., Wu, Z., Li, Z., He, Y. and Tao, W. } \\
\text { Numerical thermal optimization of the } \\
\text { configuration of multi-holed clay bricks } \\
\text { used for constructing building walls by } \\
\text { the finite volume method. }\end{array}$ & 79 & 2008 \\
\hline $21^{\circ}$ & $\begin{array}{l}\text { Antar, M.A. Thermal radiation role in } \\
\text { conjugate heat transfer across a } \\
\text { multiple-cavity building block. }\end{array}$ & 53 & 2010 \\
\hline $22^{\circ}$ & $\begin{array}{l}\text { Luo, C., Moghtaderi, B., Hands, S. and Page, } \\
\text { A. Determining the thermal capacitance, } \\
\text { conductivity and the convective heat } \\
\text { transfer coefficient of a brick wall by } \\
\text { annually monitored temperatures and } \\
\text { total heat fluxes. }\end{array}$ & 22 & 2011 \\
\hline $23^{\circ}$ & $\begin{array}{l}\text { Luo, C., Moghtaderi, B. and Page, A. } \\
\text { Modelling of wall heat transfer using } \\
\text { modified conduction transfer function, } \\
\text { finite volume and complex Fourier } \\
\text { analysis methods. }\end{array}$ & 28 & 2010 \\
\hline $24^{\circ}$ & $\begin{array}{l}\text { Boukendil, M., Abdelbaki, A. and Zrikem, Z. } \\
\text { Detailed numerical simulation of coupled } \\
\text { heat transfer by conduction, natural } \\
\text { convection and radiation through double } \\
\text { honeycomb walls. }\end{array}$ & 5 & 2012 \\
\hline $25^{\circ}$ & $\begin{array}{l}\text { Mei, F.L. and Li, G.L. Study on } \\
\text { Temperature Field and Thermal Stress for } \\
\text { an Insulated Composite Wall. }\end{array}$ & 0 & 2011 \\
\hline
\end{tabular}

Fonte: Os autores (2019). 
Construção Qualitativa sobre o Portfólio Bibliográfico com tema "Radiação Térmica em Argamassas de Revestimento"

Em relação à distribuição geográfica das publicações observa-se na figura 1 que a maioria das publicações das pesquisas em radiação térmica em argamassas de revestimento está localizada na China, Itália e Portugal, que somados representam 36\% das publicações. Em segundo lugar vem Chipre, seguido pela Grécia, México, Marrocos, Austrália e Espanha, responsáveis por 48\% das publicações nos últimos 10 anos. 0s países Índia, Noruega, República Checa e Arábia Saudita foram responsáveis por 16\% das publicações no período do estudo, sendo que cada país foi responsável por uma pesquisa no tema. No Brasil não foram encontrados trabalhos indicando que não há pesquisas sobre o este tema. $0 \mathrm{~s} 5$ primeiros artigos mais relevantes se localizam na Índia, Chipre (segundo e terceiro artigos) Itália e Noruega, respectivamente.

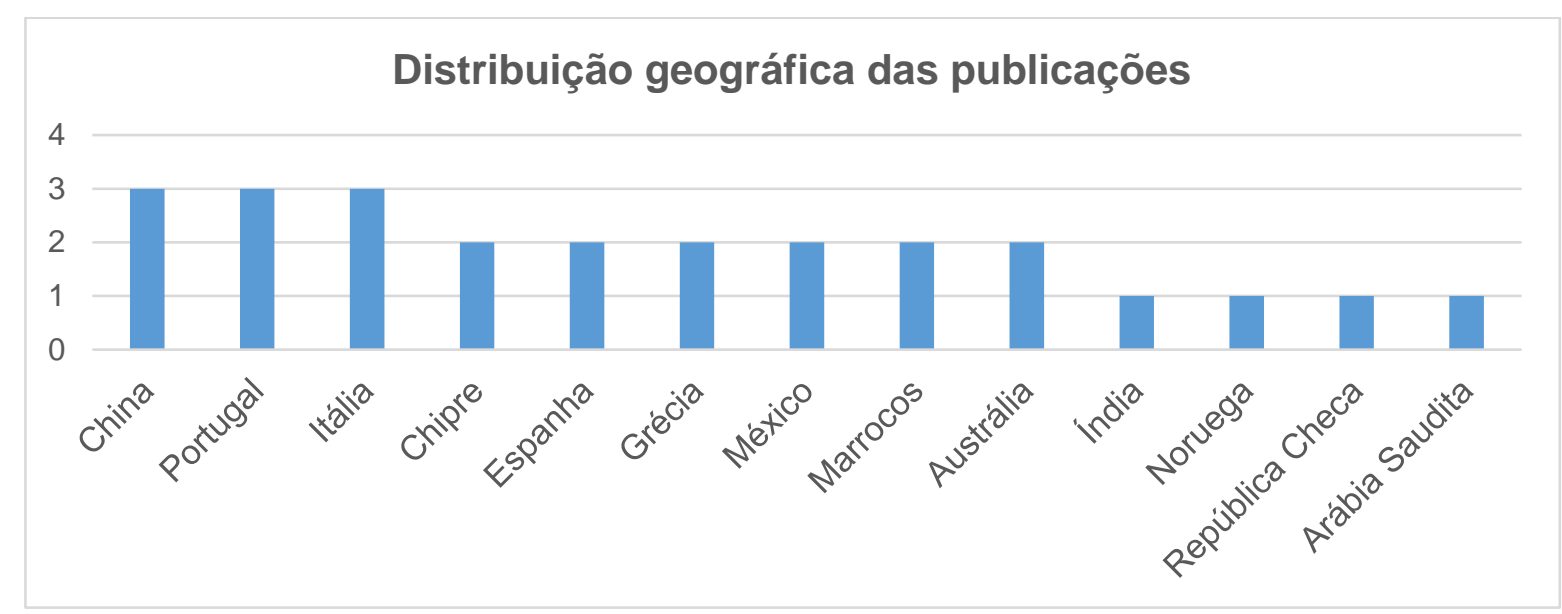

Figura 1. Localização geográfica das pesquisas de radiação térmica em argamassas de revestimento.

Fonte: 0s autores (2019).

Observa-se que $60 \%$ das publicações sobre radiação térmica em argamassas de revestimento foram feitas entre 2008 a 2016 (Figura 2). Já nos anos 2017-2018 houve um aumento nas publicações, totalizando 40\% das publicações encontradas sobre o tema, mostrando que a preocupação com a radiação térmica em argamassas de revestimento foi mais acentuada nos dois últimos anos. Embora não seja um tema recente, permanece com relevante interesse de pesquisa devido as preocupações com as questões ambientais e a melhoria da eficiência energética das construções. 


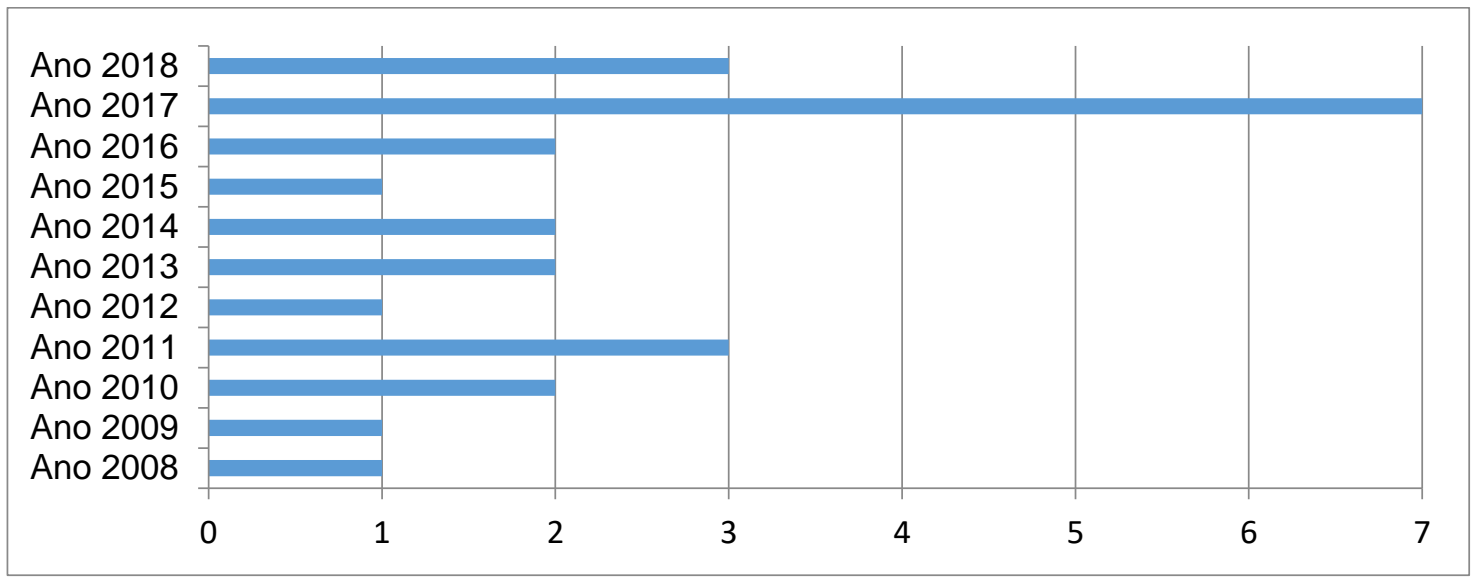

Figura 2. Distribuição das publicações por ano. Fonte: 0s autores (2019).

A revista Energy and Buildings foi a que mais publicou no tema, com 10 publicações, seguido da revista Construction and Building Materials, com 3 e Apllied Energy com 2 publicações (Tabela 4). Todas as outras revistas citadas tiveram 1 publicação, contribuindo com 4\% das publicações nesse tema nos últimos 10 anos.

Tabela 4. Número de artigos por periódico.

\begin{tabular}{lcc}
\hline \multicolumn{1}{c}{ Periódicos } & Frequência & Frequência acumulada (\%) \\
\hline Energy and Buildings & 10 & 40 \\
Apllied Energy & 3 & 12 \\
Construction and Building Materials & 2 & 8 \\
Infrared Physics e Tecnology & 1 & 4 \\
Solar Energy & 1 & 4 \\
Applied Thermal Engineering & 1 & 4 \\
Renewable and Sustainable Energy Reviews & 1 & 4 \\
Applied Mechanics and Materials & 1 & 4 \\
Indoor and Built Environment & 1 & 4 \\
Procedia Environmental Sciences & 1 & 4 \\
International Journal of Heat and Mass Transfer & 1 & 4 \\
Energy & 1 & 4 \\
Building Simulation & 1 & 4 \\
\hline
\end{tabular}

Fonte: 0s autores (2019).

0s 25 trabalhos mais relevantes são classificados em trabalhos experimentais (40\%), análise numérica (32\%) e revisão de literatura (28\%). Os trabalhos experimentais foram estudos com a finalidade de encontrar métodos de ensaios que possam abranger 0 comportamento térmico transitório de paredes que são submetidas a condições 
Construção Qualitativa sobre o Portfólio Bibliográfico com tema "Radiação Térmica em Argamassas de Revestimento"

meteorológicas reais. Na classe de análise numérica o trabalho indica o uso de modelagem de equações estruturais.

Na classe revisão de literatura foi identificada uma pesquisa sobre 0 comportamento passivo e ativo da câmera de termografia infravermelha (TRI); uma análise dos materiais que apresentam baixa emissividade e podem ser usados para reduzir o uso de energia; e por fim, uma revisão sobre os problemas recorrentes relacionados à energia (caracterização térmica de edifícios; ponte térmica, nível de isolamento, vazamento de ar e deteç̧ão de umidade; medições de temperatura interna e valor U; avaliação do conforto humano).

Diante dos dados apresentados, podemos afirmar que ainda há poucas pesquisas sobre a radiação térmica em argamassas de revestimento. No Brasil não foi encontrado nenhum artigo, demonstrando que as patologias que são causadas pela radiação térmica em argamassas de revestimento são negligenciadas no Brasil. A preocupação com a radiação térmica em argamassas de revestimento teve um aumento a partir de 2017.

Analisando os artigos selecionados frente ao desempenho térmico em edificações, observou-se que nos últimos anos, as pessoas tornaram-se conscientes dos requisitos de desempenho térmico para edifícios residenciais em regiões quentes e frias (Ramírez et al. 2018).

Aversa et al. (2017) analisa que o estudo do comportamento térmico dinâmico em condições climáticas reais dos edifícios é uma ferramenta importante para reduzir as ineficiências e desperdícios com energia. No entanto o estudo de desempenho térmico em paredes de alvenaria é de grande complexidade, verificando o efeito que todos os mecanismos de transferência de calor podem acontecer simultaneamente, como a condução através do material sólido, a propagação do calor dependente dos mecanismos de convecção e radiação na região do ar cavidades (padrão de pequenos orifícios) (KANELLOPOULOS et al. 2017).

As pesquisas também indicam um desvio entre os valores estimados na norma EN ISO 6946 (1997) e calculados em ensaios experimentais através do método de transferência de calor observados em elementos de construção (FOKAIDES; KALOGIROU, 2011). A identificação dos parâmetros de cálculo do valor-U (transmitância térmica) da norma EN ISO 6946 (1997) tem grande importância para a obtenção de resultados precisos. Os valores U obtidos são validados por meio de medições realizadas nas duas estações (verão e inverno), bem como com os resultados fornecidos pela norma EN relevante.

Aversa et al. (2017) apontam que as normas europeias especificam os procedimentos para obter informações sobre o comportamento térmico da construção em termos de fator de 
decréscimo e intervalo de tempo. Porém os estudos são baseados numa abordagem teórica que não leva em consideração os fatores reais envolvidos nos fenômenos de troca de calor, como o conhecimento correto dos parâmetros termofísicos e a presença de materiais não homogêneos ou defeitos nas paredes investigadas.

Os trabalhos selecionados apontam alguns fatores relevantes para avaliação do desempenho térmico das argamassas de revestimento, sendo eles: Irradiação Solar; Transmitância térmica (valor de U); Emissividade; Processo de secagem; Problemas de umidade; Prejuízo da umidade relativa do ar; Pontes térmicas; Medições da temperatura interna; Avaliação do conforto humano.

Cabe destacar que os materiais que compõem a parede também irão exercer influência no conforto e desempenho térmico. Jelle et al. (2015) analisaram materiais de baixa emissividade com intuito de reduzir a transferência de calor por radiação térmica para suas possíveis implementações e benefícios quando utilizados em edifícios. 0 autor conclui que há poucas pesquisas sobre o envelhecimento térmico que afeta os materiais quando são submetidos à radiação solar. 0s autores sugerem para pesquisas futuras, uma avaliação do comportamento dos materiais em relação ao envelhecimento. Mudança na emissividade devido ao envelhecimento pode alterar a eficiência energética global e uso de edifícios, e é, portanto, um fator crucial ao realizar o ciclo de vida avaliações e cálculos de energia.

Além do material a geometria de tijolos e as argamassas de revestimento, bem como as propriedades termofísicas de materiais de construção podem modificar significativamente a evolução das ondas de calor sob limite específico condições em ambos os lados externo / interno da camada examinada (ambiente) (KANELLOPOULOS et al. 2017). Além disso, a dinâmica do fluxo de calor real é afetada pelos valores de emissividade térmica nas superfícies expostas dos furos dos blocos cerâmicos, e antar (2010) concluíram que o cálculo preciso da taxa de transferência de calor através de blocos de construção pode levar significativa redução de energia elétrica.

Outro fator que influência o desempenho térmico é a espessura das argamassas (BOUKENDIL et al. 2017; B0UKENDIL et al. 2012), já que, geralmente, quanto maior a espessura, melhor o isolamento térmico. As emissividades da camada de ar e os blocos cerâmicos têm grande influência na avaliação do desempenho térmico das paredes externas, em que maiores espessuras propiciam um melhor isolamento térmico (BOUKENDIL et al. 2017; BOUKENDIL et al. 2012). 
Construção Qualitativa sobre o Portfólio Bibliográfico com tema "Radiação Térmica em Argamassas de Revestimento"

Entre as metodologias propostas nos artigos para avaliação do desempenho térmico em argamassas são indicados softwares de simulação e modelos matemáticos. Tong et al. (2009) e Kanellopoulos et al. (2017) realizaram simulações numéricas usando uma análise de dinâmica computacional de fluidos (CFD), determinado as distribuições de temperatura dependentes do tempo e a resposta térmica de paredes de alvenaria de blocos cerâmicos furados. Os resultados obtidos são úteis para promover o projeto do edifício e diminuir as incertezas em relação aos fluxos de calor através da construção de paredes. Já Luo et al. (2010) analisaram a transferência de calor por condução através da simulação numérica usando 0 método modificado de CTF, comparando com o método de elementos finitos e o método da matriz (baseado na análise complexa de Fourier). 0 método de transferência de matriz foi atualizado para tratar a radiação térmica não linear de comprimento de onda longa e mostrou-se consistente com os resultados do método finito.

Barrios et al. (2016) avaliou o desempenho térmico de paredes homogêneas e não homogêneas usando um modelo de transferência de calor dependente do tempo, através da ferramenta numérica Ener-Habitat. 0 modelo bidimensional é validado pelo cálculo do calor transferência através de uma parede de bloco de concreto em estado estacionário e a comparação com resultados experimentais. 0s resultados provaram ser uma ferramenta confiável.

Boukendil et al. (2017) estudou numericamente a transferência de calor combinada por condução, convecção natural e radiação de superfície em paredes duplas de blocos cerâmicos, através do método de elementos finitos baseado no algoritmo SIMPLE. Boukendil et al. (2012) também usaram o algoritmo SIMPLE para analisar a transferência de calor acoplada em estado estacionário por condução, convecção e radiação através de duas paredes separadas por uma camada de ar.

Tong et al. (2009), Lehman et al. (2013), Jelle et al. (2015) e Mei e Li (2011) analisaram as interações entre as condições climáticas reais e as paredes de alvenaria. Tong et al. (2009) realizaram as medições em dias de sol e verificaram a importância de analisar as paredes externas em condições climáticas nubladas sucessivas para avaliar o projeto estrutural e climático. Lehman et al. (2013) apontam a importância do estudo de vários parâmetros das construções que apresentam características distintas de desempenho térmico e de diferentes fatores de perturbação meteorológica tem influência na temperatura das paredes externas, já Jelle et al. (2015) verificam a importância de se obter valores de transmitância mais exatos para evitar o superdimensionamento de sistemas de aquecimento e resfriamento. Mei e Li 
(2011) mostraram que o isolamento externo é benéfico para a estabilidade da parede e o efeito das mudanças ambientais na fissuração das argamassas para o sistema isolado exteriormente é de grande importância.

Yang et al. (2017) estudaram o calor de convecção nas paredes externas, realizando um teste experimental de quatro estações anuais na superfície externa de uma parede através de um novo método. Os resultados obtidos através das frequências máximas durante diferentes épocas foram adquiridos com base nas estatísticas de frequência de distribuição do coeficiente de transferência de calor convectivo e transferência de calor, em um ano. As variáveis estudadas foram mudanças dinâmicas no fluxo de calor radiativo, fluxo de calor convectivo e total fluxo de calor recebido na superfície exterior em diferentes estações do ano. Os valores obtidos através do novo método foram validados analisando a influência da superfície exterior no coeficiente de transferência de calor.

Atsonios et al. (2017) estudou os métodos padronizados para a medida do valor de resistência térmica descrito em dois padrões internacionais: ISO 9869 e ASTM Cl155 e pode-se concluir que os métodos mais comumente utilizados, apresentaram grande diferença de temperatura entre as superfícies internas e externas das paredes testadas. Já Naveros et al. (2014) descreveu uma modelagem da dinâmica térmica de uma parede real para identificar todos os parâmetros necessários para sua caracterização. 0 estudo teve o objetivo de propor um método dinâmico que melhore a estimação de parâmetros que descrevem 0 comportamento térmico parede, descritos nas normas IS0 9869 e ASTM C1155. 0 valor de U obtido nesse estudo, caracterizando a parede é consistente com o dado pelo método das médias de regressão.

Briga-Sá et al. (2017) desenvolveu uma metodologia de cálculo para determinar os ganhos e perdas de fluxo de calor através por um período contínuo e o impacto do sistema de parede nos diferentes parâmetros de desempenho térmico. Com os resultados experimentais foi possível determinar a variação de temperatura, fluxo de calor, atraso térmico e velocidade do ar nas aberturas de ventilação.

Luo et al. (2011) determinaram a capacitância e condutividade térmica de uma parede empregada na construção civil através dos métodos de elementos finitos complexos de análise de Fourier, usando as temperaturas de superfície interna / externa monitorada e fluxos de calor. 0 fluxo de calor por convecção foi obtido pela remoção da radiação térmica do fluxo de calor da superfície total. 
Construção Qualitativa sobre o Portfólio Bibliográfico com tema "Radiação Térmica em Argamassas de Revestimento"

Li et al. (2008) e Antar (2010) analisaram o desempenho térmico dos blocos cerâmicos empregados em paredes de alvenaria, realizando um estudo de simulação numérica. Li et al. (2008) estudou a radiação térmica da superfície do furo, a largura e números de furos de comprimento e a diferença de temperatura interior-exterior. Já Antar (2010) analisou a interação das medidas do número de furos/ layout das cavidades cheias de ar com a taxa de transferência de calor (condução, convecção natural e radiação térmica). Li et al. (2008) concluiu que, dependendo da importância relativa de convecção natural, radiação de superfície e condução de calor através do sólido de argila, a condutividade térmica pode diminuir com 0 número de furos e aumentar de acordo com a largura. Antar (2010) concluiu que o aumento do número de cavidades mantendo a largura do bloco inalterada diminui significativamente o fluxo de calor, sendo os resultados úteis para designers e fabricantes de blocos de construção para melhor economia de energia dos usuários.

Em se tratando da análise da radiação térmica em argamassas de revestimento o método mais utilizado que foi identificado é o ensaio por termografia por infravermelho. Kylili et al. (2014), Bagavathiappan et al. (2013) e Fokaides e Kalogirou (2011) mostram a importância do método da termografia por infravermelho (IRT) para monitoramento de condições, sendo um método interessante para avaliação qualitativa das argamassas de revestimento submetidas à radiação térmica.

Segundo Lucchi (2018) as análises qualitativas se concentram em: (i) caracterização térmica de paredes, vidros e janelas; (ii) áreas de ponte térmica e de perda excessiva de calor de conveç̧ão; (iii) exame de isolamento térmico; (iv) inspeção de vazamento de ar; (v) detecção de umidade e água; (vi) e caracterização de sistemas hidráulicos e elétricos; (vii) medições de temperatura interna; e (viii) avaliação do conforto humano.

A metodologia termografia por infravermelho (IRT) é uma ferramenta útil para a caracterização de defeitos nos elementos constituintes de um edifício, no entanto, é uma técnica que precisa ser mais explorada para o desenvolvimento de abordagens mais avançadas, eficazes e mais precisas no qual pode empregar uma combinação de abordagens, sendo uma técnica que pode ser empregada em vários problemas de aplicações e ambientes de medição com uma variedade de propriedades dos materiais (FOKAIDES; KALOGIROU, 2011). Além disso, a combinação do método termografia infravermelho com métodos destrutivos, incluindo análises químicas e granulométricas, contribuiria para a caracterização e adequação das argamassas. 
A termografia por infravermelho pode ser aplicada como um diagnóstico qualitativo rápido e não destrutivo, pois as patologias se tornam visíveis através da ação de radiação solar ou uma fonte de calor, uma vez que eles criam uma camada de ar entre o revestimento e alvenaria (FREITAS et al. 2014). Pode ser empregada como uma técnica capaz de detectar problemas de umidade, desagregação de argamassas de revestimento, tijolos trincando e problemas de adesão entre os dois materiais, podendo ser útil na detecção de materiais diferentes, como por exemplo, áreas com argamassa reparada diferente da original (KYLILI et al. 2014).

Para avaliar a umidade, absorção capilar e o processo de secagem das paredes externas em escala real, Barreira et al. (2016) empregaram o método de termografia infravermelha sobre diferentes condições (período do dia e distância de visão para 0 alvo). Os autores verificaram que o método de termografia por infravermelho pode detectar problemas invisíveis de umidade e pode ser usado para avaliar o processo de secagem, embora o fenômeno traga algumas dificuldades caso se pretende realizar uma abordagem quantitativa para avaliar o desempenho térmico das paredes.

Aversa et al. (2017) propôs um procedimento experimental inovador baseado na aplicação de termografia com o objetivo de investigar o comportamento dinâmico térmico das paredes. Os resultados foram comparados com os obtidos com simulação numérica e com 0 padrão procedimento EN ISO 13786: 2007, destacando as diferenças dos valores entre as três abordagens, descritas na norma.

Lucchi (2018) analisa problemas recorrentes relacionados à energia como também vantagens e potenciais fontes de erros, bem como tendências futuras no uso de termografia por infravermelho para a auditoria energética. 0 uso de termografia por infravermelho para avaliação energética tem sido classificado em estudos qualitativos e quantitativos. 0 autor realça que o uso de termografia por infravermelho para avaliação qualitativa das edificações, tem sido bastante empregado, no entanto, para estudos quantitativos ainda estão em fase de investigação. Atualmente, concentram-se em: (i) determinação das áreas com anomalias térmicas; (ii) nível de isolamento detecção; (iii) medições do valor U e caracterização dinâmica de elementos de construção; (iv) determinação do teor de umidade; e (v) dano análise de evolução. Ainda, o autor realça os temas que necessitam de mais pesquisas: Comparação entre diferentes metodologias passivas para o edifício análise de estoque; desenvolvimento de procedimentos e ferramentas para: Medição do valor U e caracterização dinâmica do edifício componente (particularmente para paredes e sistemas de vidro); determinação da 
Construção Qualitativa sobre o Portfólio Bibliográfico com tema "Radiação Térmica em Argamassas de Revestimento"

concentração de gases em unidades de vidro e sistemas de envidraçamento; determinação do teor de umidade; deteç̧ão de evolução de dano; monitoramento ambiental de longo prazo usando o alvo simulado por termografia por infravermelho; avaliação do conforto humano; integração de diferentes testes não destrutivos para o edifício auditoria energética.

Para Tejedor et al. (2017) embora seja geralmente usado para análise qualitativa, métodos quantitativos de termografia por infravermelho também podem ser adotados para a medição in situ do valor de transmitância térmica. Os autores obtiveram valores de transmitância com um desvio de 1-2\% para paredes de camada única e 3-4\% para paredes com várias camadas.

0 modelo numérico de Tong et al. (2009) verifica a importância de analisar todos os mecanismos de transferência de calor incluindo a radiação solar variável sob temperaturas transitórias, a infiltração de ar, as capacidades térmicas das paredes espessas e a convecção natural, como também as variações de distribuição de temperatura ao longo das paredes.

Fokaides e Kalogirou (2011) acreditam que a termografia por infravermelho (IRT) apresenta a avaliação do desempenho térmico mais correta, já que o método considera também efeitos de radiação, como temperatura radiativa e a emissão das superfícies reais. Lucchi (2018) aponta a importância do método de termografia por infravermelho ser mais explorado para avaliação do desempenho térmico das edificações.

Em resumo, percebe-se que metodologias distintas são utilizadas para avaliar 0 desempenho térmico, como por exemplo, as simulações numéricas, softwares e ensaios experimentais sob condições climáticas reais. Ainda assim, as poucas pesquisas que consideram a radiação térmica na avaliação do desempenho térmico em argamassas de revestimento utilizam o método por ensaio de termografia por infravermelho para a análise da radiação térmica das edificações.

\section{CONCLUSÃ0}

Foram encontrados métodos distintos para avaliação do desempenho térmico em argamassas de revestimento, através de simulações numéricas e/ou ensaios experimentais sob condições climáticas reais.

As propriedades dos materiais de construção empregados em paredes como a geometria de blocos cerâmicos e as argamassas de revestimento influenciam significativamente na avaliação do desempenho térmico. 
A radiação térmica é negligenciada nos cálculos de desempenho térmico, não sendo considerada nas normas de muitos países. A análise da radiação térmica em paredes é de grande importância, pois analisam as condições climáticas reais de umidade, vento, processo de secagem e temperatura que influenciam no envelhecimento e no desempenho térmico.

Foram identificadas análises qualitativas com uso de termografia por infravermelho para análise do desempenho térmico. Nota-se a necessidade de desenvolver mais estudos com esta técnica para testes quantitativos para medir a real condição do desempenho térmico.

\section{REFERÊNCIAS BIBLIOGRÁFICAS}

ASSOCIAÇÃ0 BRASILEIRA DE NORMAS TÉCNICAS. NBR 15575: Desempenho de edificações habitacionais, 2013.

ANTAR, M.A. Thermal radiation role in conjugate heat transfer across a multiple-cavity building block. Energy, vol 35 , p. $3508-3516,2010$.

ATSONIOS, I.A., MANDILARAS, I.D., KONTOGEORGOS, D.A., FOUNTI, M.A. A comparative assessment of the standardized methods for the in-situ measurement of the thermal resistance of building walls. Energy and Buildings, vol 154, p. 198-206, 2017.

AVERSA, P., PALUMBO, D., DONATELLI, A., TAMBORRinO, R., ANCONA, F., GALIETTI, U. AND LUPRANO, V.A.M. Infrared thermography for the investigation of dynamic thermal behaviour of opaque building elements: Comparison between empty and filled with hemp fibres prototype wall. Energy and Buildings, vol 152, p. 264-272, 2017.

BagaVathiapPan, S., LAHIRI, B., SARAVANAN, T., PHILIP, J. AND JAYAKUMAR, T. Infrared thermography for condition monitoring - A review. Infrared Physics \& Technology, vol 60, p. 35-55, 2013.

BALAJI, N.C., MONT0, M.B.V., REDDY, V. Discerning heat transfer in building materials. Energy Procedia, vol 54, p. 654-668, 2014.

BARREIRA, E., ALMEIDA, R. AND DELGADO, J. Infrared thermography for assessing moisture related phenomena in building components. Construction and Building Materials, vol 110, p. 251-269, 2016.

BARRIOS, G., CASAS, J., HUELSZ, G. AND ROJAS, J. Ener-Habitat: An online numerical tool to evaluate the thermal performance of homogeneous and non-homogeneous envelope walls/roofs. Solar Energy, vol 131, p.296-304, 2016.

BOUKENDIL, M., ABDELBAKI, A. AND ZRIKEM, Z. Detailed numerical simulation of coupled heat transfer by conduction, natural convection and radiation through double honeycomb walls. Building Simulation, vol 5, p. 337$344,2012$.

BOUKENDIL, M., ABDELBAKI, A. AND ZRIKEM, Z. Numerical simulation of coupled heat transfer through double hollow brick walls: Effects of mortar joint thickness and emissivity. Applied Thermal Engineering, vol 125, p. 12281238, 2017.

BRIGA-SÁ, A., CUNHA, J.B., LANZINHA, J.C., PAIVA, A. Experimental and analytical approach on the trombe wall thermal performance parameters characterization. Energy and Building, vol 150, p. 265-280, 2017.

FOKAIDES, P.A. AND KALOGIROU, S.A. Application of infrared thermography for the determination of the overall heat transfer coefficient (U-Value) in building envelopes. Applied Energy, vol 88, p. 4358-4365, 2011.

FREITAS, S.S., DE FREITAS, V.P. AND BARREIRA, E. Detection of façade plaster detachments using infrared thermography - A nondestructive technique. Construction and Building Materials, vol 70, p. 80-87, 2014. 
Construção Qualitativa sobre o Portfólio Bibliográfico com tema "Radiação Térmica em Argamassas de Revestimento"

JELLE, B. P., KALNAES, S. E. AND GA0, T. Low-emissivity materials for building applications: A state-of-the-art review and future research perspectives. Energy and Buildings, vol 96, p. 329-356, 2015.

KANELLOPOULOS, G., KOUTSOMARKOS, V., KONTOLEON, K. AND GEORGIADIS-FILIKAS, K. Numerical Analysis and Modelling of Heat Transfer Processes through Perforated Clay Brick Masonry Walls. Procedia Environmental Sciences, vol 38, p. 492-499, 2017.

KIRIMTAT, A., KREJCAR, 0. A review of infrared thermography for the investigation of building envelopes: Advances and prospects. Energy and Buildings, vol 176, p. 390-406, 2018.

KYLILI, A., FOKAIDES, P.A., CHRISTOU, P. AND KALOGIROU, S.A. Infrared thermography (IRT) applications for building diagnostics: A review. Applied Energy, vol 134, p. 531-549, 2014.

LAMBERTS, R., DUTRA, L., PEREIRA, F.O.R. Eficiência Energética na Arquitetura. $3^{a}$ edição. Editora Eletrobras/Procel. Rio de Janeiro, 2014.

LEHMANN, B., WAKILI, K.G., FRANK, T., CALLADO, B.V., TAMER, C. Effects of individual climatic parameters on the infrared thermografy of building. Apllied Energy, vol 110, p. 29-43, 2013.

LI, L., WU, Z., LI, Z., HE, Y. AND TA0, W. Numerical thermal optimization of the configuration of multi-holed clay bricks used for constructing building walls by the finite volume method. International Journal of Heat and Mass Transfer, vol 5l, p. 3669-3682, 2008.

LUCCHI, E. Applications of the infrared thermography in the energy audit of buildings: A review. Renewable and Sustainable. Energy Reviews, vol 82, p. 3077-3090, 2018.

LUCCHI, E., ROBERTI, F., ALEXANDRA, T. Definition of an experimental procedure with the hot box method for the thermal performance evaluation of inhomogeneous walls. Energy and Buildings, vol 179, p. 99-111, 2018.

LUO, C., MOGHTADERI, B. AND PAGE, A. Modelling of wall heat transfer using modified conduction transfer function, finite volume and complex Fourier analysis methods. Energy and Buildings, vol 42, p. 605-617, 2010.

LUO, C., MOGHTADERI, B., HANDS, S. AND PAGE, A. Determining the thermal capacitance, conductivity and the convective heat transfer coefficient of a brick wall by annually monitored temperatures and total heat fluxes. Energy and Buildings, vol 43, p. 379-385, 2011.

MARQUES, T. H. T.; CHVATAL, K. M. S. A Review of the Brazilian NBR 15575 standard applying the simulation and simplified methods for evaluating a social house thermal performance. Symposium on Simulations for Architeture and Urban Design, 2013.

MEI, F.L. AND LI, G.L. Study on Temperature Field and Thermal Stress for an Insulated Composite Wall. Applied Mechanics and Materials, vol 100, p. 664-675, 2011.

NAVEROS, I., BACHER, P., RUIZ, D., JIMÉNEZ, M. AND MADSEN, H. Setting up and validating a complex model for a simple homogeneous wall. Energy and Buildings, vol 70, p. 303-317, 2014.

PAGANI, R., KOVALESKI, J., RESENDE, L. Methodi Ordinatio: a proposed methodology to select and rank relevant scientific papers encompassing the impact factor, number of citation, and year of publication. Scientometrics, p. $1-27,2015$.

PIZZUTI, B.R.G., ARCURI, N. The Prediction of Thermal Loads in Building by Means of the EN ISO 13790 Dynamic Model: A Comparison with TRNSYS. Energy Procedia, vol. 101, p. 192-199, 2016.

RAMÍREZ, R.A., CASTILLO, F.C., DOMÍNGUEZ, V.J.M. Thermal lag and decrement factor of constructive component reinforced mortar chamels fille whith soil-cement-sawdust. Indoor and Built Environment, vol 27, p. 466-485, 2018. 
ROELS, S.; BACHER, P.; BAWENS, G.; MADSEN, H.; JIMENEZ, M. J. Characterising the actual thermal performance of buildings current results of common exercises perfomed in the framework of the IEA EBC Annex 58 - project. Energy Procedia, vol 78, p. 3282-3287, 2015.

SASSINE, E., YOUNSI, Z., CHERIF, Y., ANTCZAK, E. Thermal performance evaluation of a massive brick wall under real weather conditions via the Conduction Transfer function method. Case studies in Construccion Materials, vol 7, p. 56-65, 2017.

TEJEDOR, B., CASALS, M., GANGOLELLS, M. AND ROCA, X. Quantitative internal infrared thermography for determining in-situ thermal behaviour of façades. Energy and Buildings, vol 151, p. 187-197, 2017.

TONG, G., CHRISTOPHER, D. AND LI, B. Numerical modelling of temperature variations in a Chinese solar greenhouse. Computers and Electronics in Agriculture, vol 68, p. 129-139, 2009.

YANG, W., ZHU, X. AND LIU, J. Annual experimental research on convective heat transfer coefficient of exterior surface of building external wall. Energy and Buildings, vol 155, p. 207-214, 2017. 